\section{Driving Voltage Reduction of a Micromechanical Optical Switch Driven by Electrostatic Force Using an S-Shaped Deformable Thin-Film Mirror*}

\author{
Kaiji SATO** \\ ** Department of Mechano-Micro Engineering, Interdisciplinary Graduate School of Science and \\ Engineering, Tokyo Institute of Technology \\ 4259-G2-17 Nagatsuta, Midori-ku, Yokohama 226-8502, Japan \\ E-mail: sato.k.ah@m.titech.ac.jp
}

\begin{abstract}
This paper describes the driving voltage reduction of an optical switch that has an S-shaped deformable thin-film mirror driven by an electrostatic force. The prototype optical switch has high-dielectric-constant layers made from $\mathrm{Ta}_{2} \mathrm{O}_{5}$ for a low applied voltage to generate a sufficiently large attractive force. Although the dielectric layers are effective at increasing the attractive force, they often cause a charging problem. Thus, suitable driving procedures for solving the charging problem were experimentally examined. In the two driving procedures, AC or DC voltage inputs were used. The experimental results indicate that the driving procedures are effective at reducing the driving voltage to $30 \mathrm{~V}$. However, the results also show that the $\mathrm{AC}$ components induce a residual vibration of the spot position. Consequently, the driving procedure with the DC voltage input is proven to be more suitable.
\end{abstract}

Key words: Optical Switch, Electrostatic Force, S Shape, Thin-Film Mirror, Dielectric Layer, Charging, Driving Voltage

\section{Introduction}

Until now, many types of micromechanical optical switches with solid micromirrors have been investigated for information systems [1-4]. Solid micromirrors provide many advantages when used in optical switches. Micromirrors are often driven by electrostatic actuators. In comparison with electromagnet actuators, electrostatic actuators can be fabricated easily as micro-electro-mechanical systems (MEMS) and produce low heat. Many electrical conducting materials can be used for the actuators. However, electrostatic actuators often require high driving voltages to produce a sufficient force. Many electrostatic actuators for optical switches are driven by a voltage above $80 \mathrm{~V}$ [5-11]. The high voltage reduces the usability of the electrostatic actuators, and this poses significant problems for many electrostatic actuators.

In past research, suitable electrode shapes have been designed for large force gains in the electrostatic actuators and reductions of the driving voltage. Comb-shaped electrodes are suitable and are often used for electrostatic actuators in MEMS devices. Optical switches with a combination of vertically positioned mirrors and horizontal comb-shaped actuators $[1,12]$, or a combination of horizontal attitude mirrors and vertical comb-shaped actuators [13] have been driven by voltages below $60 \mathrm{~V}$. However, these combinations make
*Received 11 Dec., 2012 (No. 12-0503) [DOI: 10.1299/jamdsm.7.410]

Copyright $\odot 2013$ by JSME 
the device structures complex and require bulk micromachining techniques to create deep penetrations and steep-sided holes and trenches, such as the LIGA process and deep reactive ion etching (DRIE) process. It is well known that these processes are quite expensive [14, $15]$.

The reduction of the gap between electrodes is also effective for achieving low driving voltages. When parallel electrodes are used, a small gap decreases not only the driving voltage but also the working range of the rotational mirror [16]. Sloped electrodes can relieve the problem of the working range limitation $[17,18]$. However, sloped electrodes have complex parts that require bulk micromachining, not unlike comb-shaped electrodes.

$\mathrm{S}$-shaped deformable elements have a suitable shape for low-voltage driving. The $\mathrm{S}$ shape has also been used for a novel micromechanical optical switch [19] and a microvalve $[20,21]$. Some RF switches have beam structures that offer the same advantage [22]. The $\mathrm{S}$ shape makes the gap between electrodes small at the edge, and the electrostatic force works effectively at the edge. The characteristics can be maintained across a wide working range. A microvalve has been reported to be driven by an applied voltage of $40 \mathrm{~V}$ [21]. The sloping part of an S-shaped element in a microvalve forms a small angle with the electrode that increases the driving force and reduces the required driving voltage. However, this small angle requires a wide space and is therefore not suitable for multi-channel devices. Additionally, the sloping part of the mirror in the optical switch needs to form an angle of 45 degrees with the electrodes, which is about 4.5 times greater than the angle of the film to the electrode reported in [21].

This paper describes the driving voltage reduction of a micromechanical optical switch using an S-shaped deformable thin-film mirror. In this research, a high-dielectric-constant material is used to increase the electrostatic force gain and to decrease the minimum driving voltage. This method is considered to be useful for devices requiring a large angle such as multi-channel devices. However, it generally causes a significant charging phenomenon that greatly deteriorates the performance and reliability of the electrostatic actuators [23, 24]. The phenomenon and its repression have often been discussed [25-27]. To repress the negative effects of charging, suitable driving signals must be determined.

This paper is organized as follows: The principles of the micromechanical optical switch with the S-shaped mirror are introduced in Section 2. Section 3 explains the concept of the driving voltage reduction method. In Section 4, the fabrication process and a prototype of the switches are shown. Section 5 discusses the effects of driving procedures with alternating current (AC) and direct current (DC) voltage inputs on the driving characteristics based on experiments. Concluding remarks are then presented in Section 6.

\section{Principle}

Figure 1 shows the principle of the micromechanical optical switch with an S-shaped thin-film mirror [19]. In the figure, the case of a $1 \times 4$ switch is illustrated. The core unit of this switch consists of two substrates, which are set parallel to each other, and an S-shaped conductive thin-film mirror located between the substrates. The film mirror between the substrates is set to form an angle of 45 degrees with the substrates for bending light rays at a right angle. At least one of the substrates and its electrodes are made from transparent materials so that light rays can pass through the substrate. The substrates and electrodes that are located under the layer are coated with an insulation layer.

When a voltage is applied between the film mirror and the electrodes in the substrates, the electrostatic force causes the film to stick to the substrates. Using this force, the film mirror between the substrates is set to form an angle of 45 degrees with the substrates. The arrows in the figure indicate that the electrostatic force is produced between the film and the electrode. In Fig. 1(a), the incident light ray parallel to the substrates is reflected by the 
$\Omega$ Voltage differing from that of the mirror

Voltage equal to that of the mirror

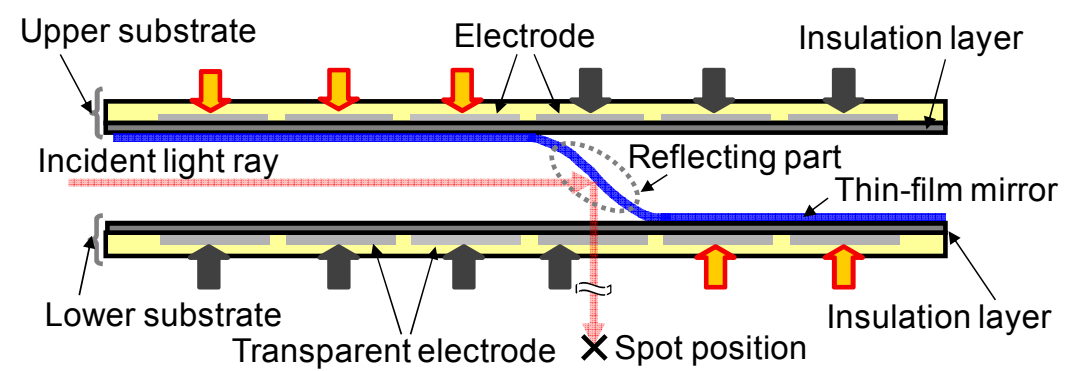

(a) Relationship between spot position and reflecting part located by the applied voltage pattern

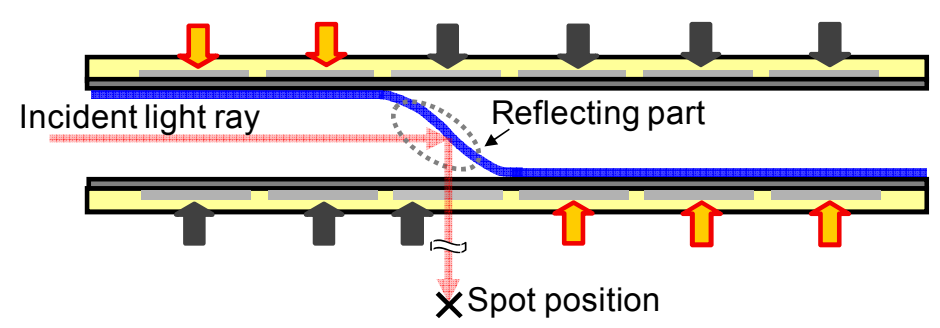

(b) Movement of the spot position to the left by changing the voltage pattern

Fig. 1 Principle of micromechanical optical switch using an S-shaped thin-film mirror

thin-film mirror between the substrates and passes through the lower substrate. When the voltage pattern applied to the electrodes is changed as shown in Fig. 1(b), the position of the sloping part of the film mirror (reflecting part) and the spot position of the reflected light ray move to the left side; that is, the spot position is switched. Since the optical switch outputs the light ray out of the plane, it can be used as a scan line for displays and scanners in addition to components of $\mathrm{N} \times \mathrm{N}$ optical switches [19].

\section{Reduction Method for Driving Voltage}

The $\mathrm{S}$ shape leads to a small gap between the film mirror and the electrode at the edge of the mirror part not in contact with the substrates, and the electrostatic force works effectively at the edge. Figure 2 shows the S-shaped thin-film mirror near the edge. When the S-shaped thin-film mirror is driven, the attractive force produced near the edge of the reflecting part very close to the substrate greatly influences the minimum driving voltage. In Area (A) of Fig. 2, where the thin-film mirror faces the electrode, the electrostatic force between a small part of the mirror and the electrode $\Delta f_{a e}$ can be approximately expressed as

$$
\Delta f_{a e}=\frac{\varepsilon_{0}}{\varepsilon_{a}} \cdot \frac{w \Delta x}{2\left(\frac{t_{a}}{\varepsilon_{a}}+\frac{t_{i}}{\varepsilon}\right)^{2}} V_{i n}{ }^{2}
$$

where

$V_{\text {in }}:$ Applied voltage

$\varepsilon_{0}$ : Vacuum permittivity 
$\varepsilon_{a}:$ Relative permittivity of air

$\varepsilon$. Relative permittivity of insulation layer

$w$ : Width of mirror

$\Delta x$ : Small length of mirror

$t_{\mathrm{a}}$ : Air gap between mirror part and substrate

$t_{\mathrm{i}}$ : Thickness of insulation layer

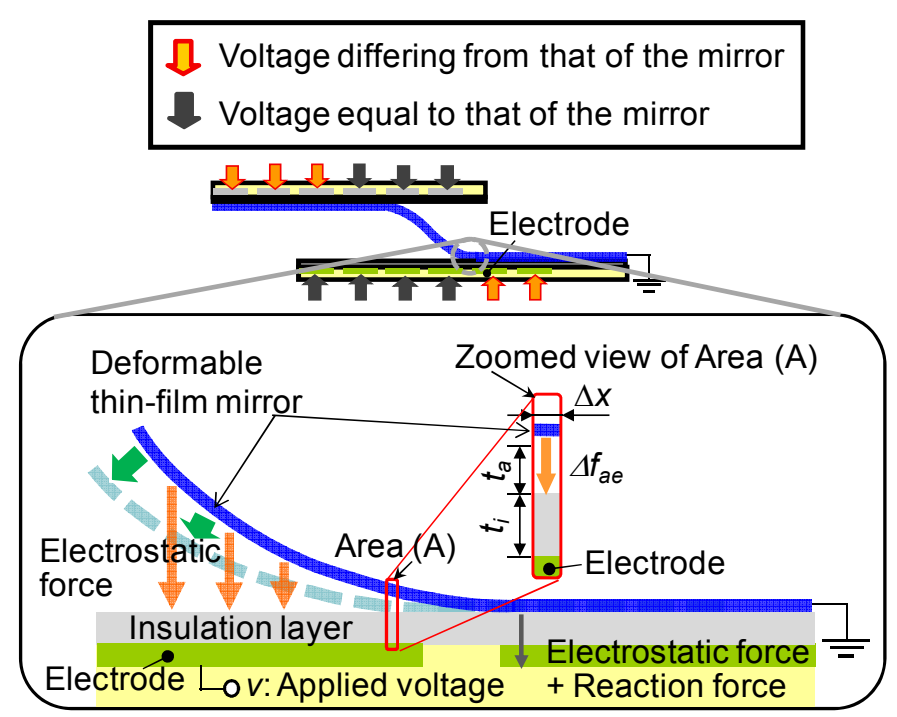

Fig. 2 Behavior of S-shaped thin-film mirror near the edge of the mirror part not in contact with the substrates

This equation indicates that the use of a high-dielectric-constant material for the insulation layer increases the attractive electrostatic force. Its use is effective at reducing the minimum driving voltage and does not influence the dimensions of the switch. However, the high-dielectric-constant layer often causes a significant charging phenomenon that greatly deteriorates the response and reliability of the electrostatic actuators. Since the actuator performance is important, a method for suppressing such charging needs to be developed.

The charging phenomenon is caused by the DC component of the driving voltage and is suppressed by the AC component of the driving voltage. Thus, driving using an AC component is effective at suppressing the charging. In Section 5, the usefulness of the AC driving voltage is discussed. Aside from this, the addition of a step for the change of the applied voltage direction will be useful for eliminating the negative charging effect. The usefulness of this addition is also examined experimentally in that section.

\section{Prototype Core Switch}

\subsection{Fabrication of Components}

Figure 3 shows a 3-D schematic diagram of the prototype optical core switch fabricated in this study. The prototype was constructed and used to examine the driving characteristics with a high-dielectric-constant insulation layer. The core switch consists of three components: an upper substrate, a lower substrate, and an S-shaped deformable thin-film mirror. The electrodes in the substrates are arranged in a line. The core switch works as a $1 \times \mathrm{N}$ switch. The incident rays also move along the electrode array.

Figure 4 shows the fabricated substrate used as the upper substrate and lower substrate in Fig. 3 and its structure. The upper and lower substrates have the same structure. They were fabricated using photolithography because of its accessibility. Transparent glass was used as the base material. The electrodes were constructed from InTiO so that light rays can 


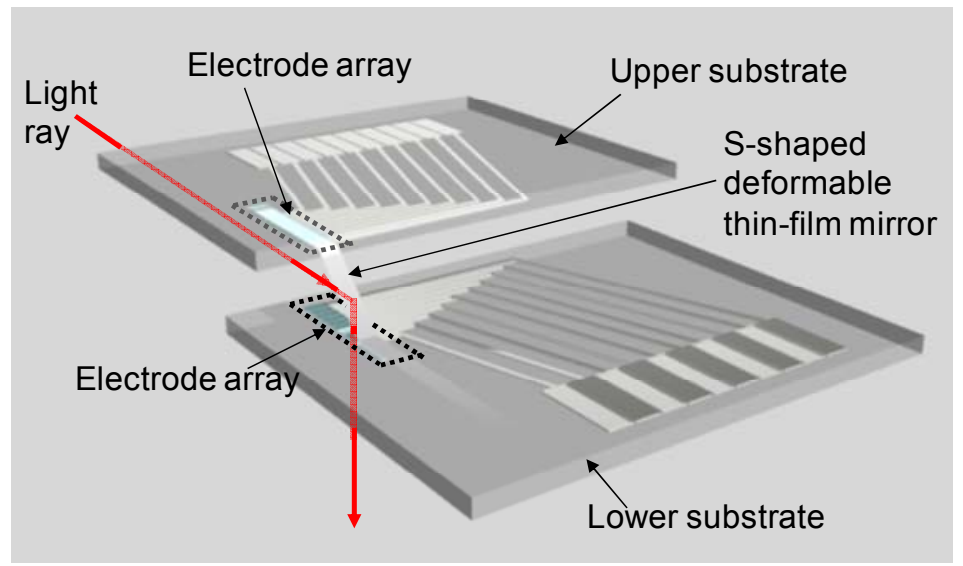

Fig. 3 3-D schematic diagram of the prototype optical core switch

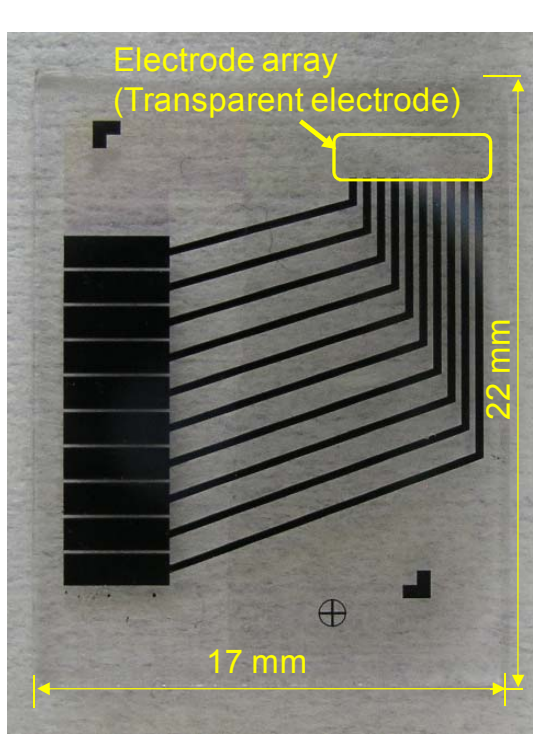

(a) Fabricated substrate

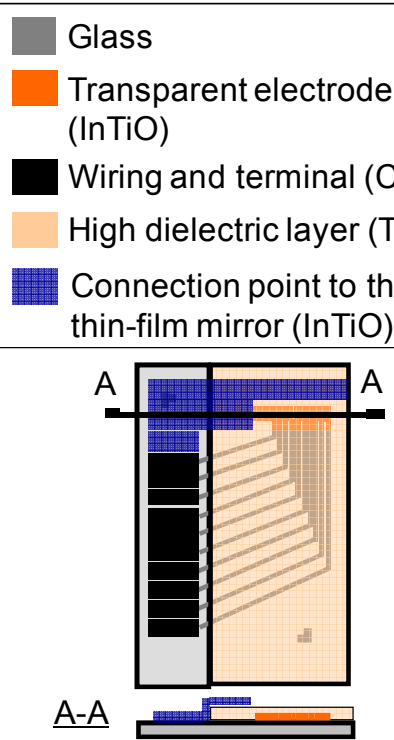

(b) Structure

Fig. 4 Fabricated substrate of the core switch

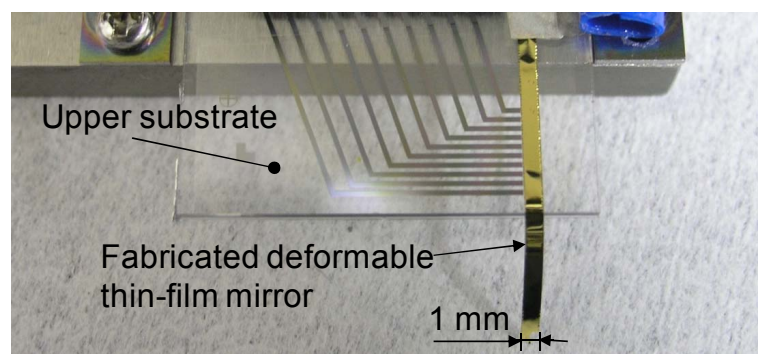

Fig. 5 Fabricated deformable thin-film mirror attached on the upper substrate

pass through the substrate. For easy wiring, $\mathrm{Cr}$ (indicated by the dark areas) was used as a visible conductor material. Although the insulation layers in the previous prototype optical switches were made from $\mathrm{SiO}_{2}$ [19], those in this prototype were made from $\mathrm{Ta}_{2} \mathrm{O}_{5}$, whose dielectric constant is about six times larger than that of $\mathrm{SiO}_{2}$. Figure 5 shows the fabricated deformable thin-film mirror attached to the upper substrate. In this figure, the mirror surface for reflecting light rays is shown. This mirror is used as the S-shaped deformable thin-film mirror shown in Fig. 3. The fabricated mirror is constructed from Ti-Ni alloys, which have 


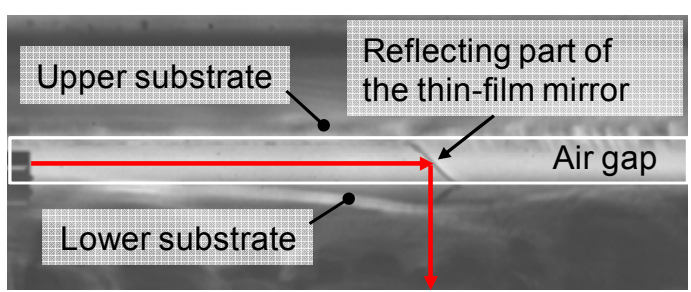

(a) Close-up view of the prototype core switch

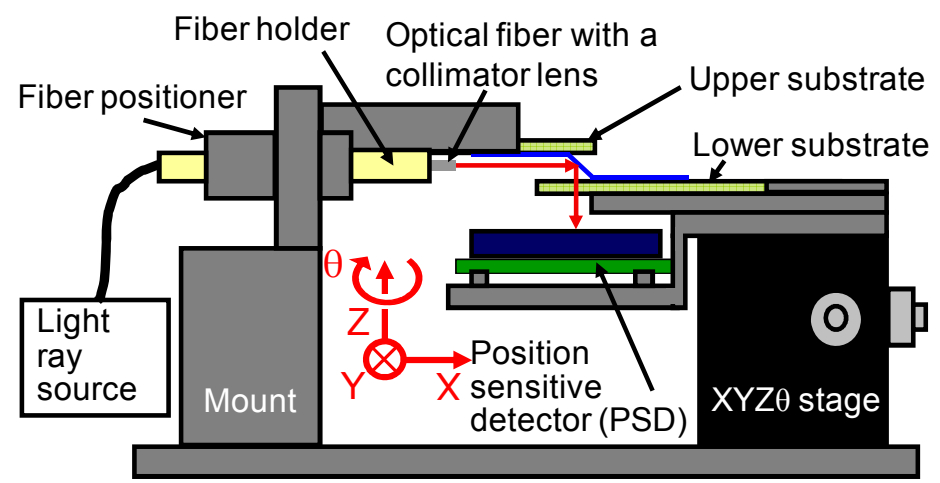

(b) Overview of experimental setup

Fig. 6 Experimental setup

a large allowable deformation and a high fatigue strength. Two mirrors with different Ti-Ni alloy thicknesses $(0.5 \mu \mathrm{m}$ and $0.3 \mu \mathrm{m})$ were manufactured. An Au layer $(50 \mathrm{~nm})$ was applied to the alloys and used as the mirror surface.

\subsection{Setting}

Figure 6 shows the prototype core switch constructed from the fabricated components and the experimental setup including the core switch. A photograph of the prototype core switch from a lateral view is shown in Fig. 6(a). First, the thin-film mirror was bonded to the upper substrate using an electrically conductive adhesive. Next, the gap between the substrates was adjusted, and the center angle of the reflecting part of the mirror was adjusted to be 45 degrees to the substrates. A gap of $0.3 \mathrm{~mm}$ was used.

The setting process was performed using the experimental setup illustrated in Fig. 6(b). The experimental setup comprises an XYZ $\theta$ fine stage, a light source on a mount, and a position sensitive detector (PSD). The upper and lower substrates are positioned on the mount and the fine stage, respectively. The fine stage is used to adjust the components. The light source emits light rays through a $0.25-\mathrm{mm}$ diameter lens. The driving characteristics are examined using light with a $670 \mathrm{~nm}$ wavelength because this allows easy operation. The spot position of the light rays reflected by the thin-film mirror is measured using the PSD to examine the characteristics of the core switches.

\section{Experimental Discussion}

Figure 7 shows the basic driving procedure for moving the reflecting part one step to the left, which is used in [19]. The voltage pattern in Fig. 7(a) makes the angle of the reflecting part 45 degrees, as shown in Fig. 1. For one pitch motion of the reflecting part, the voltage patterns in Figs. 7(b) and (c) are applied to the electrode array in sequence. The voltage pattern in Fig. 7(c) makes the angle of the reflecting part 45 degrees again. The basic driving procedure in Fig. 7 with a DC voltage input can drive the prototype core switch in Fig. 6 for a while, but charging limits the driving time. In this section, the effect of 
the two applied driving procedures on the driving voltage reduction is discussed; that is, the driving procedure in Fig. 7 with sinusoidal AC voltage inputs and an improved driving procedure having new voltage patterns with DC voltage inputs.

\subsection{Effect of driving procedure with AC voltage inputs}

In order to avoid charging the substrates, sinusoidal AC voltages were applied to the electrodes with the voltage input in Fig. 7, and their effects were examined experimentally. In the experiments, two mirror thicknesses $(0.35$ $\mu \mathrm{m}$ and $0.55 \mu \mathrm{m}$ ) and three sinusoidal $\mathrm{AC}$ voltage inputs with different frequencies $(100 \mathrm{~Hz}, 1 \mathrm{kHz}$, and $10 \mathrm{kHz})$ were used.

Figure 8 shows the one-pitch $(0.5 \mathrm{~mm})$ stepwise response of the light spot using the prototype core switch with a mirror thickness of $0.55 \mu \mathrm{m}$. The dimensions of the core switch and the applied voltage are shown at the top of Fig. 8. Leftward movement of the spot in Fig. 1 is defined as positive movement. The amplitude and frequency of the

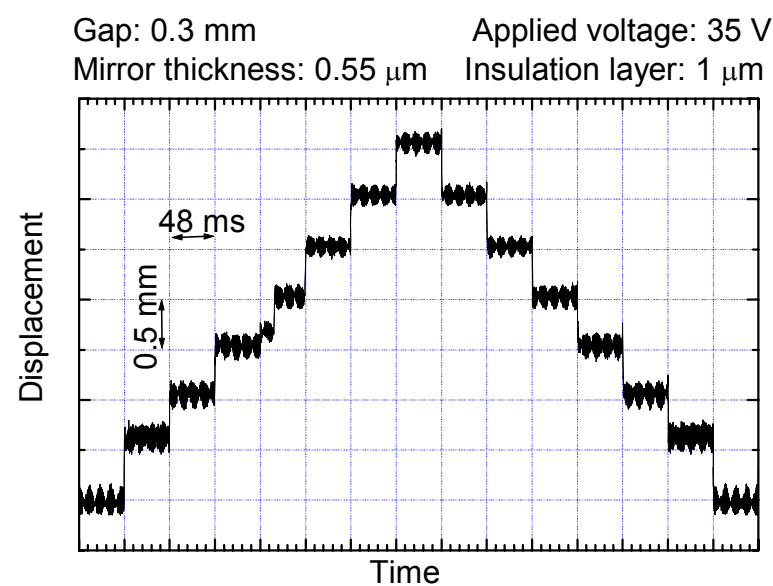

Fig. 8 Stepwise response of the light spot using the basic driving procedure with AC voltage input (Frequency: $1 \mathrm{kHz}$ )

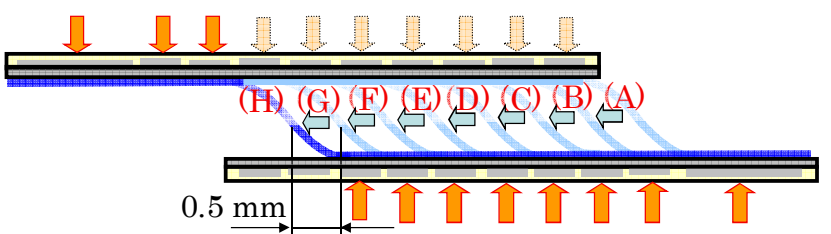

Fig. 9 Definition of the mirror position

applied voltage are $35 \mathrm{~V}$ and $1 \mathrm{kHz}$, respectively. The switching period for each step height is $48 \mathrm{~ms}$. During this period, the time for the transient state shown in Fig. 7(b) (transient-state time) is $0.75 \mathrm{~ms}$. Figure 9 shows the definition of the mirror position. In the 


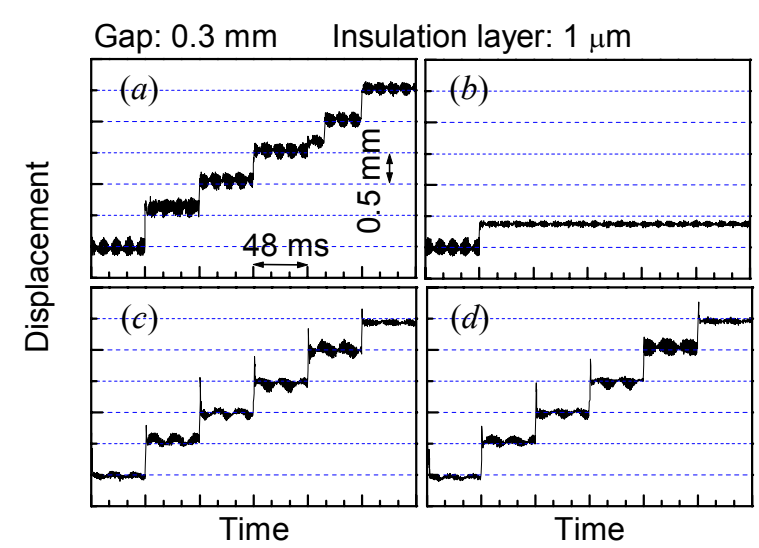

(a) Mirror thickness of $0.55 \mu \mathrm{m}$, Amplitude of $35 \mathrm{~V}$

(b) Mirror thickness of $0.55 \mu \mathrm{m}$, Amplitude of $30 \mathrm{~V}$

(c) Mirror thickness of $0.35 \mu \mathrm{m}$, Amplitude of $35 \mathrm{~V}$

(d) Mirror thickness of $0.35 \mu \mathrm{m}$, Amplitude of $30 \mathrm{~V}$

Fig. 10 Effect of input voltage amplitude on the response using a $1 \mathrm{kHz}$ sinusoidal voltage input

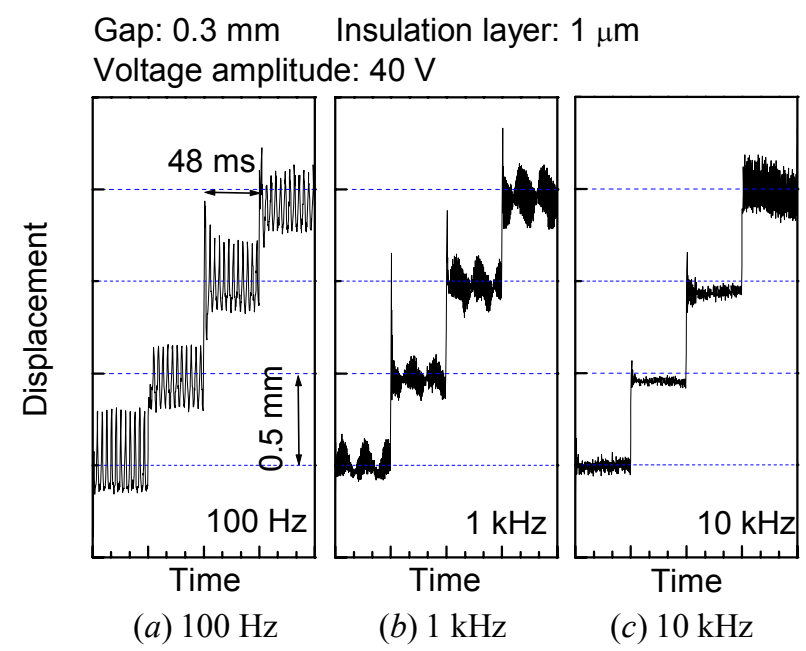

Fig. 11 Frequency effect on response

experiment, the reflecting part of the mirror was moved from point (A) to $(\mathrm{H})$ and from point $(\mathrm{H})$ to $(\mathrm{A})$ one step at a time. The change of the spot position per period becomes approximately one pitch, i.e., $0.5 \mathrm{~mm}$. In this study, the response of the spot position with respect to the initial spot position is referred to as the displacement. A sinusoidal AC voltage input of $35 \mathrm{~V}$ amplitude successfully moves the average spot position of the core switch with the high-dielectric-constant insulation layers to the target position, although the spot position vibrates. This peak-to-peak (p-p) driving voltage is smaller than the minimum driving voltage of $130 \mathrm{~V}$ shown in [19].

The effect of the input voltage amplitude on the response is shown in Fig. 10 using a 1 $\mathrm{kHz}$ sinusoidal voltage input. The dimensions of the core switch are shown at the top of the figure. An amplitude of $35 \mathrm{~V}$ can steadily drive the core switches with $0.35-\mu \mathrm{m}$ and $0.55-\mu \mathrm{m}$ thick mirrors. However, an amplitude of $30 \mathrm{~V}$ can drive only the $0.35-\mu \mathrm{m}$ thick mirror.

The response of the spot position is greatly influenced by the frequency of the input voltage. The frequency effect on the response is shown in Fig. 11. A voltage input of $100 \mathrm{~Hz}$ 
causes significant vibration of the spot position. The vibration amplitude decreases with an increase of the frequency. However, the steady-state vibration is not completely eliminated. The vibration reduction is important for driving the switch using AC voltage.

\subsection{Improvement of the driving procedure with DC voltage inputs}

In order to overcome the charging problem and reduce the steady-state vibration, the driving procedure was improved by using a DC voltage input. Figure 12 shows the improved driving procedure. The voltage pattern to the electrodes is changed from (a) to (b) to (c) to (d) to (e). In the procedure, the voltage inputs to the electrodes near the edge of the reflecting part are changed to move the reflecting part. The changes from (a) to (b) and from (d) to (e) are performed to eliminate the charging problem. This procedure is expected to effective discharge the insulation layer just prior to the switching motion of the mirror. The use of DC voltage input can stably maintain the mirror position.

Figure 13 shows the experimental one-pitch stepwise response of the spot position with the driving procedure of Fig. 12. As in Fig. 8, the reference spot position was switched. The relationship bety ween the spot position and the reference point is shown on the left side of the figure. The dimensions of the core switch and the applied voltage are shown at the top of the figure. The period for each step height is $8 \mathrm{~ms}$. For each

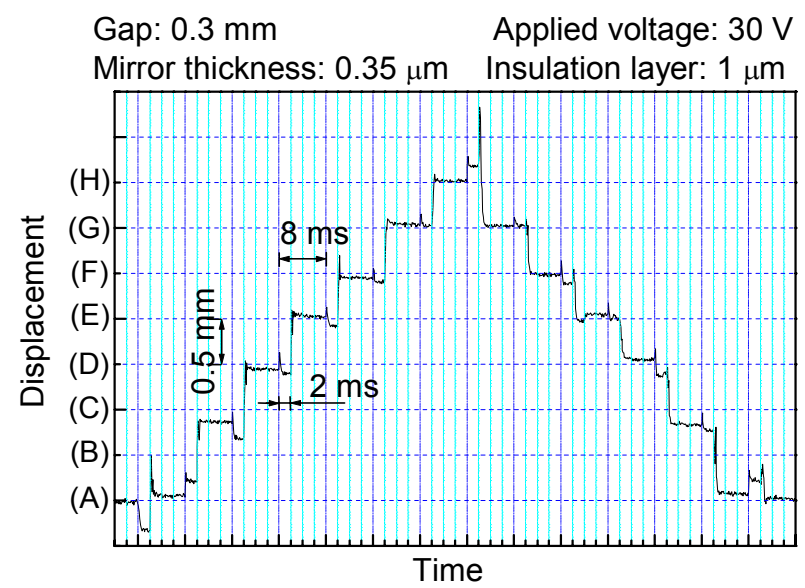

Fig. 13 Stepwise response of light ray spot using the improved driving procedure with DC voltage input 


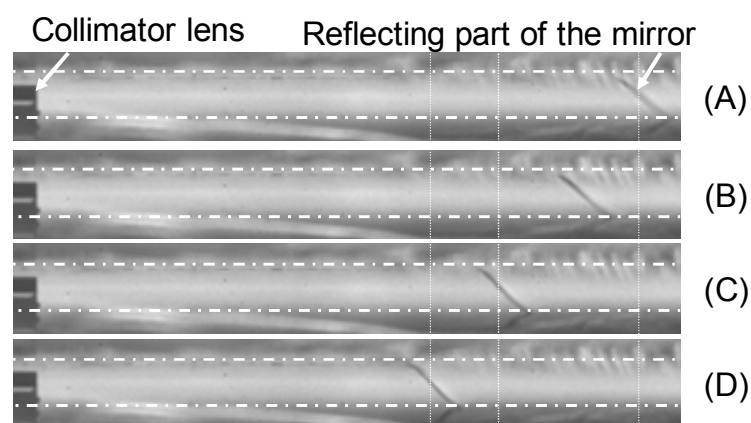

Fig. 14 Behavior of the reflecting part from Position (A) to (D).

voltage pattern, it takes $2 \mathrm{~ms}$. Voltage pattern (b) causes a transient response of the spot position. Voltage patterns (c), (d), (e) and (a) provide a stable spot position. Although the spot position command for point (B) did not provide sufficient displacement, the spots are positioned precisely at the other points. The result indicates that an amplitude of $30 \mathrm{~V}$ is effective enough for switching the spot. Although a voltage input of $25 \mathrm{~V}$ could move the reflecting part, the motion range was limited. This driving procedure provides a similar minimum driving voltage without the steady-state vibration to the driving procedure with an AC voltage input presented in Section 5.1. On the whole, this shows a better performance than the driving procedure in Section 5.1.

In order to examine the behavior of the reflecting part at position (B), the behavior was measured with a high-speed video camera. Figure 14 shows side-view photographs of the reflecting part after the step command inputs for the four positions (A), (B), (C), and (D). All the step commands produce significant step motion. However, the distance between positions (A) and (B) is shorter than the other distances. In addition, the angle between the reflecting part and the substrates at position (B) is similar to those at the other points. This is considered to be caused by fabrication problems and results in a large spot position error for the reference input of position (B).

\section{Conclusion}

In this paper, the driving voltage reduction of an optical switch driven by an electrostatic force using an S-shaped thin film has been discussed. To achieve this reduction, dielectric layers and suitable driving procedures were used and their usefulness was experimentally evaluated. Since the dielectric layers cause a charging problem, a driving procedure with $\mathrm{AC}$ voltage inputs and an improved driving procedure with $\mathrm{DC}$ voltage inputs were applied to the prototype core switches. The experimental results show that the driving procedure with an $\mathrm{AC}$ voltage input of $30 \mathrm{~V}$ can drive the switch. However, AC voltage inputs always cause the spot position to vibrate. In contrast, the improved driving procedure with a DC voltage of $30 \mathrm{~V}$ was proven to drive the switch without residual spot vibration. These results will prove useful for many devices with S-shaped thin-film elements driven by an electrostatic force.

The improved driving procedure with a $\mathrm{DC}$ voltage input of $25 \mathrm{~V}$ provides a stepwise motion, although its working range is smaller than that with a DC voltage input of $30 \mathrm{~V}$. This suggests that minimizing fabrication errors can decrease the minimum driving voltage. Thus, the plan for future work is to improve the fabrication process.

This paper focused on driving voltage reduction, not on spot position error. The spot position error in Fig. 13 is considered to result from fabrication errors. Thus, improvement of the fabrication process is important for the reduction of the spot position error. In contrast to the driving procedure with $\mathrm{AC}$ voltage inputs, the improved driving procedure does not produce residual vibration. This has a positive effect on the position error of an optical 
switch with a low fabrication error. Conventional optical switches are often required to have a spot position error smaller than $2 \mu \mathrm{m}$. Reducing position error is a future goal, although the allowable spot position error depends on the specifications of the receiver units.

\section{Acknowledgments}

The author would like to thank Mr. M. Nishimura for his help in obtaining the experimental results reported in this paper. This work was supported in part by the Casio Science Promotion Foundation.

\section{References}

[1] Marxer, C. and de Rooij, F. N., Micro-Opto-Mechanical 2 x 2 Switch for Single-Mode Fibers Based on Plasma-Etched Silicon Mirror and Electrostatic Actuation, IEEE/OSA Journal of Lightwave Technology, Vol. 17, No. 1 (1999), pp. 2-6.

[2] Syms, R. R. A., Scaling Laws for MEMS Mirror-Rotation Optical Cross Connect Switches, IEEE/OSA Journal of Lightwave Technology, Vol. 20, No. 7 (2002), pp. 1084-1095.

[3] Cheng, Y. C., Dai C. L., Lee C. Y., Chen P. H. and Chang P. Z., A Circular Micromirror Array Fabricated by a Maskless Post-CMOS Process, Microsystem Technologies, No. 11 (2005), pp. 444-451.

[4] Sadat, S. H., Kamiya, D., Bagheri, S. and Horie, M., 2 Degree-of-Freedom Spiral Micromirror Manipulator Fabrication and Spatial Mechanical Assembling, Journal of Advanced Mechanical Design, Systems, and Manufacturing, Vol. 2, No. 2 (2008), pp. 265-270.

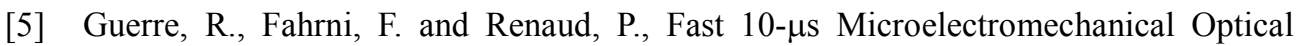
Switch inside a Planar Hollow Waveguide (PHW), IEEE/OSA Journal of Lightwave Technology, Vol. 24, No. 3 (2006), pp. 1486-1498.

[6] Lin, L. Y., Goldstein, E. L. and Tkach, R. W., Free-space Micromachined Optical Switches with Submillisecond Switching Time for Large-Scale Optical Crossconnects, IEEE Photonics Technology Letters, Vol. 10, No. 4 (1998), pp. 525-527.

[7] Lee, S. S., Huang, L. S., Kim, C. J. and Wu, M. C., Free-Space Fiber-Optic Switches Based on MEMS Vertical Torsion Mirrors, IEEE/OSA Journal of Lightwave Technology, Vol. 17, No. 1 (1999), pp. 7-13.

[8] Toshiyoshi, H. and Fujita, H., Electrostatic Micro Torsion Mirrors for an Optical Switch Matrix, IEEE/ASME Journal of Microelectromechanical Systems, Vol. 5, No. 4 (1996), pp. 231-237.

[9] Greywall, D. S., Busch, P. A., Pardo, F., Carr, D. W., Bogart, G. and Soh, H. T., Crystalline Silicon Tilting Mirrors for Optical Cross-Connect Switches, IEEE/ASME Journal of Microelectromechanical Systems, Vol. 12, No. 5 (2003), pp. 708-712.

[10] Chu, P. B., Brener, I., Pu, C., Lee, S.-S., Dadap, J. I., Park, S., Bergman, K., Bonadeo, N. H., Chau, T., Ming C., Doran, R. A., Gibson, R., Harel, R., Johnson, J. J., Lee, C. D., Peale, D. R., Bo T., Tong, D. T. K., Tsai, M.-J., Wu, Q., Zhong, W., Goldstein, E. L., Lin, L.Y.; and Walker, J.A., Design and Nonlinear Servo Control of MEMS Mirrors and Their Performance in a Large Port-Count Optical Switch, IEEE/ASME Journal of Microelectromechanical Systems, Vol. 14, No. 2 (2005), pp. 261-273.

[11] Wang, Z. F., Cao, W., Shan, X. C., Xu, J. F., Lim, S. P., Noell, W. and de Rooij, N. F., Development of 1 x 4 MEMS-Based Optical Switch, Sensor and Actuators A, Vol. 114, (2004), pp. 80-87.

[12] Kwon, H. N. and Lee, J. H., A Micromachined 2 × 2 Optical Switch Aligned with Bevel-Ended Fibers for Low Return Loss, IEEE/ASME Journal of 
Microelectromechanical Systems, Vol. 13, No. 2 (2004) pp. 258-263.

[13] Kim, M., Park, J. H., Jeon, J. A., Yoo, B. W., Park, I. H. and Kim, Y. K., High Fill-Factor Micromirror Array Using a Self-Aligned Vertical Comb Drive Actuator with Two Rotational Axes, Journal of Micromechanics and Microengineering, Vol. 19, (2009), p. 035014 (9pp).

[14] Takimoto, S., Ueno, H., Sugiyama, S., Yamaguchi, M., Baba, M. and Arai, K., Fabrication of Thick Film Magnetic Cores for High Frequency Using LIGA Process, IEEE Transactions on Magnetics, Vol. 37, No.4 (2001), pp. 2888-2890.

[15] Yang, Y. J. and Liao, B. T., A Novel 4 x 4 Optical Switch Using an Anisotropically Etched Micromirror Array and a Bistable Mini-Actuator Array, IEEE Photonics Technology Letters, Vol. 21, No. 2 (2009) pp. 115-117.

[16] Sun, D. M., Dong, W., Wang, G. D., Liu, C. X., Yan, X., Xu, B. K. and Chen, W. Y., Study of a $2 \times 2$ MOEMS Optical Switch with Electrostatic Actuating, Sensor and Actuators A, Vol. 120, No. 1 (2005) pp. 249-256.

[17] Sun, D., Dong, W., Guo, W., Liu, C., Wang, G., Yan, X., Xu, B., Chen, W. and Kraft, M., Study on Actuating Voltage and Switching Time of a MOEMS Optical Switch, Optics \& Laser Technology, No. 37 (2005), pp. 601-607.

[18] Jin J. Y., Park, J. H., Yoo, B. W. and Jang, Y. H., Numerical Analysis and Demonstration of a 2-DOF Large-Size Micromirror with Sloped Electrodes, Journal of Micromechanics and Microengineering, Vol. 21, (2011), p. 095006 (12pp).

[19] Sato, K. and Okutsu, K., Basic Characteristics of a Micromechanical Optical Switch Using an S-Shaped Deformable Thin-Film Mirror, IEEE/OSA Journal of Lightwave Technology, Vol. 29, No. 18 (2011), pp. 2805-2811.

[20] Shikida, M., Sato, K., Tanaka, S., Kawamura, Y. and Fujisaki, Y., Electrostatically Driven Gas Valve with High Conductance, IEEE/ASME Journal of Microelectromechanical Systems, Vol. 3, No. 2 (1994), pp. 76-80.

[21] Sato, K. and Shikida, M., An Electrostatically Actuated Gas Valve with an S-Shaped Film Element, Journal of Micromechanics and Microengineering, Vol. 4, No. 4 (1994), pp. 205-209.

[22] Chu, C. H., Shih, W. P., Chung, S. Y., Tsai, H. C., Shing, T. K. and Chang, P. Z., A Low Actuation Voltage Electrostatic Actuator for RF MEMS Switch Applications, Journal of Micromechanics and Microengineering, Vol. 17, (2007), pp. 1649-1656.

[23] Mardivirin, D., Pothier, A. E. I., Khatib, M., Crunteanu, A., Vendier, O. and Blondy, P., Reliability of Dielectric Less Electrostatic Actuators in RF-MEMS Ohmic Switches Proceedings of European Microwave Conference 2008, (2008-10), pp. 490-493.

[24] Bochobza-Degani, O., Socher, E. and Nemirovsky, Y., On the Effect of Residual Charges on the Pull-in Parameters of Electrostatic Actuators, Sensors and Actuators A Vol. 97-98, (2002), pp. 563-568.

[25] Fukushige, T., Hata, S. and Shimokohbe, A., A MEMS Conical Spring Actuator Array, IEEE/ASME Journal of Microelectromechanical Systems, Vol. 14, No. 2 (2005), pp. 243-253.

[26] Rottenberg, X., De Wolf, I., Nauwelaers, K. J. C., De Raedt, W. and Tilmans, H. A. C., Analytical Model of the DC Actuation of Electrostatic MEMS Devices with Distributed Dielectric Charging and Nonplanar Electrodes, IEEE/ASME Journal of Microelectromechanical Systems, Vol. 16, No. 5 (2007), pp. 1243-1253.

[27] van Spengen, W. M., Puers, R. and De Wolf, I., The Prediction of Stiction Failures in MEMS, IEEE Transactions on Device and Materials Reliability, Vol. 3, No. 4 (2003), pp. 167-172. 